\title{
Hydroxyl safflower yellow A regulates the tumor immune microenvironment to produce an anticancer effect in a mouse model of hepatocellular carcinoma
}

\author{
YICONG MA ${ }^{1,2^{*}}$, CUILING FENG $^{3 *}$, JINGJING WANG $^{1,4}$, ZIWEI CHEN $^{1}$, PENG WEI $^{1}$, ANGRAN FAN $^{1}$, \\ XU WANG ${ }^{1}$, XUE YU $^{1}$, DONGYU GE ${ }^{1}$, HUA XIE $^{1}$, LI LIU $^{1,5}$, QIAN ZHANG ${ }^{1}$ and XU-HUI LI ${ }^{2}$ \\ ${ }^{1}$ Institute of Traditional Chinese Medicine, Beijing University of Chinese Medicine, Beijing 100029; \\ ${ }^{2}$ Zhejiang Provincial Key Laboratory of Applied Enzymology, Yangtze Delta Region Institute of Tsinghua University, \\ Jiaxing, Zhejiang 314006; ${ }^{3}$ Department of Traditional Chinese Medicine, Peking University People's Hospital, \\ Beijing 100044; ${ }^{4}$ Oncology Microstart Intervention Department, Anyang Hospital of Traditional \\ Chinese Medicine, Anyang, Henan 455000; ${ }^{5}$ Institute of Chinese Materia Medica, China \\ Academy of Chinese Medical Sciences, Beijing 100700, P.R. China
}

Received March 29, 2018; Accepted November 29, 2018

DOI: $10.3892 / 01.2019 .9946$

\begin{abstract}
Hepatocellular carcinoma (HCC) is a serious threat to human health. Chemotherapy drugs such as cisplatin are widely used in cancer treatment, but can cause severe side effects. Hydroxyl safflower yellow A (HSYA) is a water-soluble chalcone glycoside substance extracted from safflowers (Carthamus tinctorius L.) that has been reported to inhibit tumor growth with few side effects. The tumor immune microenvironment is crucial for the proliferation and invasiveness of tumor cells, and it is mediated by forkhead box P3-positive $\left(\mathrm{FOXP}^{+}\right)$regulatory $\mathrm{T}$ cells (Tregs) and retinoic acid receptor-related orphan receptor- $\gamma(\mathrm{ROR} \gamma)$-expressing Th17 cells. FOXP3 ${ }^{+}$Tregs inhibit immunoreaction and FOXP3 is a key indicator of Tregs. ROR $\gamma$ isoform 2, also known as ROR $\gamma \mathrm{t}$, is an important transcription factor in Th17 cells that may promote cancer progression. In the present study, the antitumor effect of HSYA on HCC was investigated, as well as its impact on the tumor immune microenvironment. Following the establishment of a mouse model for HCC, hematoxylin and eosin staining were performed to observe histological changes
\end{abstract}

Correspondence to: Professor Qian Zhang, Institute of Traditional Chinese Medicine, Beijing University of Chinese Medicine, 6 Yinghuayuan Street, Chaoyang, Beijing 100029, P.R. China E-mail: zq19881988@163.com

Dr Xu-Hui Li, Zhejiang Provincial Key Laboratory of Applied Enzymology, Yangtze Delta Region Institute of Tsinghua University, 705 Yatai Street, Jiaxing, Zhejiang 314006, P.R. China

E-mail: lixuhui07@mails.tsinghua.edu.cn

*Contributed equally

Key words: hydroxyl safflower yellow A, hepatocellular carcinoma, regulatory $\mathrm{T}$ cell, tumor immune microenvironment, cisplatin in liver tumors, and the spleen and thymus were weighed to calculate the spleen and thymus indexes. The proportion of FOXP $3^{+}$Tregs in the spleen was determined by flow cytometry, and expression levels of Foxp3 and Ror $\gamma t$ were examined by reverse transcription-quantitative polymerase chain reaction and western blot analysis. The results of the present study showed that cisplatin inhibited tumor growth, caused weight loss and reduced the immunoreactivity of the mice. HSYA inhibited tumor growth without causing significant weight loss. The proportion of FOXP3-expressing Tregs in the spleen and the expression of Foxp3 and Ror $\gamma t$ mRNA decreased following treatment with certain doses of HSYA. In conclusion, HSYA inhibited tumor growth without detrimental effects on the weight of the mice, indicating that HSYA may be suitable as a novel therapy for HCC patients.

\section{Introduction}

Hepatocellular carcinoma (HCC) is one of the most common types of cancer of the digestive system. HCC is the third most common cause of cancer-associated mortality worldwide (1-4), and the second in China (5). Surgery is the preferred treatment for HCC, while chemotherapy is applied in cases of unresectable $\mathrm{HCC}(6)$. A poor prognosis and high mortality rate continue to be associated with HCC. The tendency of intrahepatic metastasis in the early stages means that HCC is frequently not identified while it remains resectable (7). Damage to the immune system following radiotherapy and chemotherapy further increases the difficulty in the rehabilitation of patients (8).

Cancer is determined not only by the genetic composition of the tumor cells, but also by the surrounding environment, which supports the growth, proliferation and metastasis of tumor cells (9). While a significant body of research has focused on the characteristics and mechanisms of HCC cells, the role of the tumor microenvironment in $\mathrm{HCC}$ is largely unknown. Certain immune cells in the tumor microenvironment directly promote the growth of tumor cells. Macrophages 
can produce insulin-like growth factor-1 to activate the extracellular signal-regulated kinase and protein kinase B pathways in lung cancer, directly stimulating tumor proliferation and growth (10). Moreover, regulatory T cells (Tregs) can significantly increase the invasive ability of cancer cells and inhibit the antitumor immune response in ovarian cancer (11). The extent of forkhead box P3-positive (FOXP3 ${ }^{+}$) Treg infiltration in HCC tissue is associated with high-grade, poorly differentiated tumors, and may be indicative of a poor prognosis (12). Th17 is a type of helper $\mathrm{T}$ cell produced by differentiation from Th0 cells upon stimulation with interleukin (IL)-6 and IL-23. Th17 cells secrete proinflammatory factors, including IL-17 and IL-22; and retinoic acid receptor-related orphan receptor- $\gamma$ (ROR $\gamma$ ), especially the isoform ROR $\gamma 2$ (also known as ROR $\gamma \mathrm{t}$ ), is an important transcription factor in this process (13). High expression of IL-17 and IL-17 receptors is associated with a poor prognosis in patients with HCC (14).

Cisplatin is one of the most commonly used and effective anticancer chemotherapy drugs, but it is associated with severe tissue damage and other adverse reactions (15). Previous studies have indicated that cisplatin has higher efficacy and fewer side effects when used in combination with other anticancer drugs (16). Moreover, herbs from traditional Chinese medicines have been reported to improve the immune response of patients with HCC (17).

Herbs, including safflower (Carthamus tinctorius L.), have been demonstrated to promote blood circulation and remove blockages to treat menstrual disorders, trauma, joint pain and abdominal masses in traditional Chinese medicine $(18,19)$. The main active component of safflower is hydroxyl safflower yellow A (HSYA). Recently, HSYA has been found to attenuate the apoptosis of peripheral blood $\mathrm{T}$ lymphocytes positive for T-cell surface glycoprotein CD4 (CD4) in a murine model of sepsis, to inhibit the proliferation and metastasis of tumor cells and tumor angiogenesis, and to induce tumor cell apoptosis (20-24). Furthermore, HSYA treatment has been demonstrated to decrease the expression of matrix metalloproteinase and hypoxia inducible factor $1 \alpha$ in mice with xenograft tumors $(25,26)$.

In the present study, the potential anticancer effect of HSYA on the tumor immune microenvironment in HCC was investigated. Following the establishment of an HCC model in BALB/c mice, the anticancer effect of HSYA at varying concentrations was evaluated, the Treg ratio in the spleen was assessed and the expression of several crucial molecules in the tumor microenvironment was measured. We hypothesized that HSYA treatment would have anticancer effects and low toxicity in mouse $\mathrm{HCC}$, highlighting the potential of this compound in cancer therapy.

\section{Materials and methods}

Reagents. HSYA (purity $\geq 95 \%$ ) was purchased from China Pharmaceutical and Biological Products (Beijing, China). Cisplatin was purchased from Sigma-Aldrich; Merck KGaA (Darmstadt, Germany; product number, P4394; concentration $\leq 100 \%$ ). fetal bovine serum (FBS) was obtained from Hyclone; GE Healthcare Life Sciences (Logan, UT, USA), high glucose-DMEM from Gibco; Thermo Fisher Scientific, Inc., (Waltham, MA, USA) and the Mouse Regulatory T Cell
Staining kit from eBioscience; Thermo Fisher Scientific, Inc. The SV Total RNA Isolation system and SYBR Green mix were obtained from Promega Corporation (Madison, WI, USA), the primers from Sangon Biotech Co., Ltd. (Shanghai, China), the anti-FOXP3 (cat. no. ab75763) and anti-ROR $\gamma \mathrm{t}$ (cat. no. ab207082) antibodies from Abcam (Cambridge, MA, USA) and the SuperEnhanced chemiluminescence detection kit from Applygen Technology, Inc. (Beijing, China).

Animals and cells. A total of 70 male BALB/c mice, aged 6-8 weeks, weighing 19-21 g each, were purchased from Si Pei Fu Laboratory Animal Technology Co. Ltd. (Beijing, China; certificate no: SCXK Jing 2011-0004). The mice were raised in the animal room of Beijing University of Chinese Medicine, at $25^{\circ} \mathrm{C}$ with $12 \mathrm{~h}$ of light and $12 \mathrm{~h}$ of darkness, and fed with food and water. The Hepa1-6 murine HCC cell line was purchased from the Institute of Basic Medical Sciences (Chinese Academy of Medical Sciences, Beijing, China). Cells were maintained in high-glucose DMEM supplemented with $10 \% \mathrm{FBS}$ at $37^{\circ} \mathrm{C}$ in a humidified atmosphere containing 5\% $\mathrm{CO}_{2}$. Following 3 to 4 passages, cells in the logarithmic growth phase were used for the subsequent experiments.

Model establishment. The experiment was approved by the Institutional Animal Ethics Committee of Beijing University of Chinese Medicine (Beijing, China). A PBS suspension was made of Hepa1- 6 cells at $4 \times 10^{7}$ cells $/ \mathrm{ml}$. BALB/c mice were acclimated to their surroundings for 7 days and fasted for $12 \mathrm{~h}$. Anesthesia was then induced by isoflurane inhalation $(2-4 \%)$ and preoperative subcutaneous application of buprenorphine $(0.1 \mathrm{mg} / \mathrm{kg})$ (27). Following anesthesia, the mice were placed on a plate in the supine position and their fur was removed. Subsequent to sterilization with iodine, a laparotomy was performed along the linea alba, exposing the abdominal cavity. Light pressure was applied to the thoracic cavity to expose the mouse liver. A microsyringe was used to inject $1 \times 10^{6}$ Hepal- 6 cells into the surface of the proximal body of the liver, while the mice in the control group were injected with an equal volume of normal saline. The syringe tip was inserted $\sim 1 \mathrm{~cm}$ into the liver at a $15^{\circ}$-angle. A total of $25 \mu \mathrm{l}$ of the cell suspension was injected slowly. The needle was slowly withdrawn, pressure was applied for a moment with a sterile cotton swab and then the liver was reinserted into the abdominal cavity. The abdomen was closed and the wound was disinfected. All mice were sacrificed 11 days later by cervical dislocation. Following laparotomy, a tumor could be observed in the liver, indicating the success of establishing the model.

Animal grouping. In total, 70 mice were randomly divided into 7 groups $(n=10)$. Subsequent to receiving an injection of Hepa1-6 cells (saline for the control group) into the liver on day 1, the mice were treated as follows: The control group and model group were injected intraperitoneally with $0.2 \mathrm{ml}$ normal saline twice per day. The high-, medium- and low-dose groups received intraperitoneal injections of HSYA at 2.25, 1.13 and $0.57 \mathrm{mg} / \mathrm{kg}$ twice per day, respectively. These doses were selected using preliminary experimental results. The cisplatin group received an intraperitoneal injection of $5 \mathrm{mg} / \mathrm{kg}$ cisplatin once every 2 days at a fixed time in the morning, and 
the combination group received an intraperitoneal injection of $0.57 \mathrm{mg} / \mathrm{kg}$ HSYA twice per day, plus $5 \mathrm{mg} / \mathrm{kg}$ cisplatin once every 2 days. After 11 days, all mice were sacrificed.

Histological examination. Tumors were excised and fixed in $4 \%$ paraformaldehyde for $48 \mathrm{~h}$ at room temperature, rinsed with water, dehydrated with gradient ethanol and embedded in paraffin at $56^{\circ} \mathrm{C}$. The slices were cut into $4-\mu \mathrm{m}$ layers and stained with hematoxylin for $5 \mathrm{~min}$ and subsequently, with eosin for $1 \mathrm{~min}$ at room temperature. Pathological changes to the tumor tissue was observed under an optical microscope (Olympus Corporation, Tokyo, Japan).

Weight and index calculation. The body weights of the mice were recorded every 2 days. Subsequent to sacrifice, the spleen and thymus were aseptically removed and weighed, and the spleen and thymus indexes were calculated as follows: Spleen index $=$ spleen weight $(\mathrm{mg}) /$ mouse weight $(\mathrm{g})$ x 10 ; and thymus index=thymus weight $(\mathrm{mg}) /$ mouse weight $(\mathrm{g}) \times 10$. The spleen and thymus index reflect the weight of the spleen and thymus, which are related to the number of immune cells contained within. An increasing index indicates enhanced immunity (28).

Flow cytometry. The Treg ratio in the spleen was assessed by flow cytometry according to the protocol of the Mouse Regulatory T Cell Staining kit. Briefly, a suspension of spleen cells $\left(1 \times 10^{7}\right.$ cells $\left./ \mathrm{ml}\right)$ was prepared by grinding, filtering and washing the spleen samples with PBS. A total of $100 \mu \mathrm{l}$ cell suspension was added to each flow tube. Anti-mouse CD4 $(0.25 \mu \mathrm{l})$ and anti-mouse IL-2 receptor subunit $\alpha$ CD25 (CD25) $(0.3 \mu \mathrm{l})$ antibodies were added and incubated for $60 \mathrm{~min}$ at $4^{\circ} \mathrm{C}$. The cells were then washed and lysed. Fc block $(1 \mu \mathrm{l})$ was added and incubated for $15 \mathrm{~min}$ at room temperature. Next, anti-mouse FOXP3 antibody $(2.0 \mu \mathrm{l})$ was added. Following incubation at room temperature for $60 \mathrm{~min}$, the cells were washed and analyzed using a flow cytometer (BD Biosciences, San Jose, CA, USA). FlowJo software, version 10 (FlowJo LLC, Ashland, OR, USA) was used to analyze the sample data.

Reverse transcription quantitative-polymerase chain reaction analysis (RT-qPCR). Total RNA was extracted from tumor samples using the SV Total RNA Isolation system. The reverse transcription reaction was performed as follows: Firstly, the reaction mixture (3 $\mu \mathrm{g}$ total RNA, Oligo (dT), nuclease-free water) was incubated at $95^{\circ} \mathrm{C}$ for $5 \mathrm{~min}$, and immediately placed on ice; secondly, the RT reaction was performed at $42^{\circ} \mathrm{C}$ for $60 \mathrm{~min}$, and at $75^{\circ} \mathrm{C}$ for $15 \mathrm{~min}$ in a $20 \mu \mathrm{l}$ volume with $3 \mu \mathrm{g}$ total RNA, dNTPs, Oligo (dT), RNasin ribonuclease inhibitor, Moloney murine leukemia virus (M-MLV) reverse transcriptase, $5 \mathrm{X}$ reverse transcriptase buffer and nuclease-free water. qPCR was performed with a CFX96 Real Time-PCR system (Bio-Rad Laboratories, Inc., Hercules, CA, USA) in a $20 \mu 1$ reaction volume of $2 \mu \mathrm{l} \mathrm{cDNA}, 1 \mu \mathrm{l}$ primer, $7 \mu \mathrm{l}$ nuclease-free water and $10 \mu \mathrm{l} \mathrm{SYBR}$ Green mix. The sequences of the PCR primers were as follows: Foxp3 forward, 5'-CTGCCTTGG TACATTCGTGAAC-3' and reverse, 5'-ATGTTGTGGGTG AGTGCTTTG-3'; Ror $\gamma t$ forward, 5'-GCTCTGCCAGAATGA CCAGA-3' and reverse, 5'-CAGCTCCACACCACCGTATT-3'; and $\beta$-actin forward, 5'-CCAGCCTTCCTTCTTGGGTATG-3' and reverse, 5'-TGTTGGCATAGAGGTCTTTACGG-3'. PCR was performed as follows: Denaturation at $95^{\circ} \mathrm{C}$ for $10 \mathrm{~min}$, followed by 39 cycles at $95^{\circ} \mathrm{C}$ for $15 \mathrm{sec}, 55^{\circ} \mathrm{C}$ for $30 \mathrm{sec}$ and $72^{\circ} \mathrm{C}$ for $30 \mathrm{sec}$. Melting curve analysis was performed to measure the specificity of the PCR products. Expression was calculated using the $2^{-\Delta \Delta \mathrm{Cq}}$ method (29), relative to $\beta$-actin.

Western blot analysis. Protein was isolated from tumor tissue by routine methods with radioimmunoprecipitation assay buffer (Beijing Solarbio Science \& Technology Co., Ltd., Beijing, China) and quantified using the BCA method. The protein was separated by $10 \%$ SDS-PAGE (30 $\mu$ g protein/lane), and transferred to polyvinylidene fluoride membranes (EMD Millipore, Bedford, MA, USA). The membranes were blocked with $5 \%$ skimmed milk [ $1 \mathrm{~g}$ skimmed milk powder in $20 \mathrm{ml}$ TBS with $1 \%$ Tween (TBST)] overnight. The membrane was then incubated with unconjugated rabbit anti-mouse primary antibodies against $\mathrm{FOXP}^{+}$and $\mathrm{ROR} \gamma \mathrm{t}$ at $4^{\circ} \mathrm{C}$ overnight (anti-bodies were diluted by TBST at 1:1,000 with 1\% sodium azide), washed with TBST 3 times (10 min each), incubated with a HRP-conjugated goat-anti-rabbit IgG secondary antibody (cat. no. 7090; Abcam, Cambridge, MA, USA) for $1 \mathrm{~h}$ at room temperature and washed again. Finally, the bands were visualized using the SuperEnhanced chemiluminescence detection kit.

Statistical analysis. The experimental data are expressed as the mean \pm standard deviation. Multigroup comparisons were performed using one-way analysis of variance and the least significant difference post hoc test. All statistical analyses were performed with SPSS 20.0 statistical software (IBM Corporation, Armonk, NY, USA). P $<0.05$ was considered to indicate a statistically significant difference.

\section{Results}

Effect of HSYA on tumorigenesis and pathological changes in HCC model mice. HE staining was performed to detect pathological changes in the livers of the mice, particularly to observe any alterations in tumor growth. Liver cells of the mice from the control group displayed red-stained circular cytoplasm and blue-stained nuclei, the liver lobular structural integrity was highly ordered, and the largest vein of the liver was cord-like and positioned centrally (Fig. 1A). A large area of solid tumor tissue was found in the central vasculature in the model group, with tumor cells occurring in clumps (Fig. 1B). Tumor cells were generally smaller and more deeply stained than the normal adjacent cells. The HSYA high- (Fig. 1C), middle- (Fig. 1D) and low- (Fig. 1E) concentration groups, and the cisplatin group (Fig. 1F) all exhibited scattered cancer cell clumps. The number of cancer cells was markedly lower in these groups than in the model group, and only precancerous pathological changes were observed. The liver lobular structural integrity of the mice in the cisplatin group was disordered. In the combination group (Fig. 1G), scattered liver cancer cells and evidence of precancerous pathological alterations were observed, but the normal structure of the liver cells was more intact. Therefore, HSYA inhibited the proliferation of liver cancer cells and reduced the extent of tissue damage induced by cisplatin. 

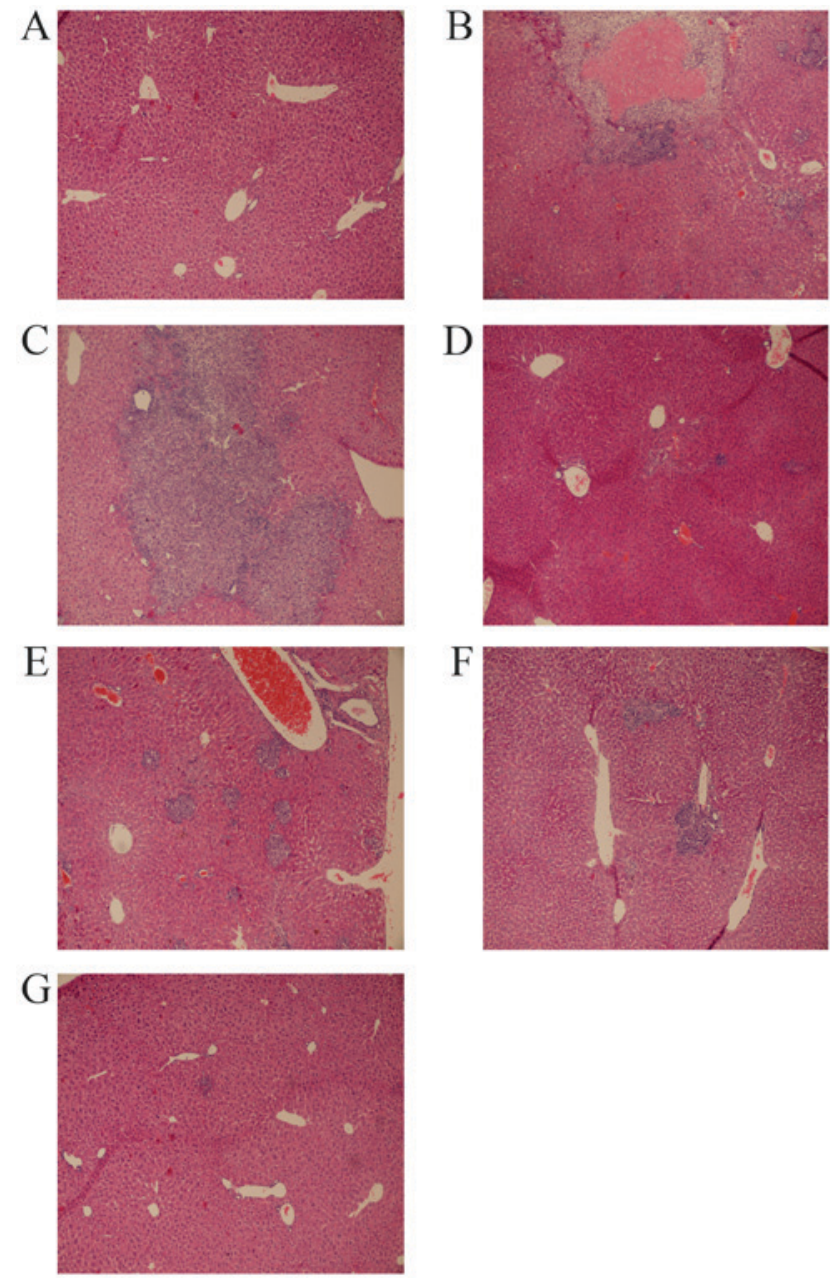

Figure 1. Pathological changes to mouse liver tissue in hepatocellular carcinoma model mice. (A) The control group was treated with saline intraperitoneally for 11 days follwing injection of saline into the liver. All other groups received an injection of Hepa1-6 cells into the liver, followed by: (B) saline (model group); (C) $2.25 \mathrm{mg} / \mathrm{kg} \mathrm{HSYA} \mathrm{(high-dose} \mathrm{group);}$ (D) $1.13 \mathrm{mg} / \mathrm{kg}$ HSYA (medium-dose group); (E) $0.57 \mathrm{mg} / \mathrm{kg}$ HSYA (low-dose group); (F) $5 \mathrm{mg} / \mathrm{kg}$ cisplatin (cisplatin group); or (G) $0.57 \mathrm{mg} / \mathrm{kg}$ HSYA and $2.5 \mathrm{mg} / \mathrm{kg}$ cisplatin (combination group). Liver tissues were excised and stained with hematoxylin and eosin to view pathological changes (magnification, x100). HSYA, hydroxyl safflower yellow A.

Effect of HSYA on body weight of HCC model mice. In order to examine whether HSYA induced adverse effects on mice or reduced the side-effects caused by cisplatin, the body weight of the mice was measured every 2 days. As shown in Table I, the mice treated with cisplatin or a combination of cisplatin and HSYA exhibited a decreasing trend in body weight, while mice treated with HSYA alone gained weight. Compared with that of the control group, the body weight of the model group increased, which may have been caused by tumor growth. The body weight of the HSYA groups was not significantly different to the body weight of the model group, while the weight of the mice in the cisplatin group was significantly decreased from the 5th day compared with that of the model group $(\mathrm{P}<0.01)$. The weight of the mice in the combination group also decreased from the 7 th day $(\mathrm{P}<0.01)$. Compared with the cisplatin group, the body weight of the mice in the combination group was significantly higher on the 5th day $(\mathrm{P}<0.05)$. These results indicate that HSYA treatment did not lead to negative effects on mouse body weight and reduced the extent of weight loss in the mice treated with cisplatin.

Effect of HSYA on the thymus and spleen indexes in HCC model mice. To investigate whether HSYA protected the immune system from damage by cisplatin treatment, the thymus and spleen indexes were measured as indicators for immunity. The effect of HSYA on the thymus and spleen indexes is displayed in Fig. 2. Compared with the control group, the thymus and spleen indexes of the model group decreased $(\mathrm{P}>0.05)$, whereas compared with the model group, the HSYA groups exhibited an increased thymus index $(\mathrm{P}<0.05)$. The effects of middle $(1.13 \mathrm{mg} / \mathrm{kg})$ and low $(0.57 \mathrm{mg} / \mathrm{kg})$ concentrations of HSYA on the thymus index were particularly significant $(\mathrm{P}<0.01)$. However, as for the spleen index, compared with the control group, there was no significant change in the model group and compared with the model group, there was no significant change in the HSYA groups. In the cisplatin and combination groups, the thymus and spleen indexes were significantly decreased $(\mathrm{P}<0.01)$. Compared with the cisplatin group, the combination group trended towards an increase in the thymus and spleen indexes, but there was no significant difference. These results indicated that HSYA treatment increased the thymus index of HCC model mice, potentially reflecting an improvement in immunity, whereas HSYA could not rescue the decreased thymus index induced by cisplatin treatment.

Effect of HSYA on the proportion of $\mathrm{CD}^{+} \mathrm{CD}_{25} 5^{+} \mathrm{FOXP} 3^{+}$ Tregs in the spleen of HCC model mice. As the aforementioned data indicates, HSYA inhibited tumor growth and improved the thymus index. The underlying mechanisms were then considered. As illustrated in Fig. 3, the ratio of $\mathrm{CD}^{+} \mathrm{CD} 25^{+} \mathrm{FOXP} 3^{+}$ Tregs to all $\mathrm{CD}^{+}{ }^{+} \mathrm{T}$ lymphocytes $\left(\mathrm{CD} 4^{+} \mathrm{CD} 25^{+} \mathrm{FOXP} 3^{+}\right.$ Tregs/CD4 ${ }^{+}$Tlymphocytes) in the spleen of mice from the model group was significantly higher than that of the control group $(\mathrm{P}<0.01)$. The ratio of $\mathrm{CD} 4^{+} \mathrm{CD} 25^{+} \mathrm{FOXP} 3^{+}$Tregs in the spleen was significantly decreased in mice treated with $1.13 \mathrm{mg} / \mathrm{kg}$ HSYA compared with the model group $(\mathrm{P}<0.05)$, whereas the ratio change was not significant for the high $(2.25 \mathrm{mg} / \mathrm{kg})$ and low $(0.57 \mathrm{mg} / \mathrm{kg})$ HSYA groups. These results indicated that HSYA may decrease the proportion of Tregs in the spleen of HCC model mice at specific doses. This may be because Tregs only respond to HSYA at a certain concentration range, but this conclusion requires further investigation.

Effect of HSYA on the expression of Foxp3 and Roryt mRNA in tumor tissue from HCC model mice. The expression levels of Foxp3 and Ror $\gamma t$ mRNA in tumor samples are displayed in Fig. 4. The expression of Foxp3 $(\mathrm{P}<0.05)$ and $\operatorname{Ror} \gamma t(\mathrm{P}<0.01)$ was increased in the model group compared with that in the control group. Compared with that in the model group, the expression of Foxp3 was decreased in the high- and medium-dose HSYA groups, particularly in the middle group $(1.13 \mathrm{mg} / \mathrm{kg})(\mathrm{P}<0.01)$. Ror $\gamma t$ expression was decreased in the medium-dose group only $(\mathrm{P}<0.01)$ compared with that in the model group. These results indicated that HSYA treatment reduced the expression of Foxp3 and Ror $\gamma t$ mRNA at certain doses.

Effect of HSYA on FOXP3 and ROR $\gamma$ t protein expression in tumor tissue from HCC model mice. Compared with that 
Table I. Effect of HSYA on body weight of hepatocellular carcinoma model mice.

\begin{tabular}{lcccccc}
\hline Group & 1 day & 3 days & 5 days & 7 days & 9 days & 11 days \\
\hline Control & $20.19 \pm 1.001$ & $20.91 \pm 1.660$ & $21.18 \pm 1.885$ & $21.46 \pm 1.773$ & $21.23 \pm 2.119$ & $21.23 \pm 2.356$ \\
Model & $20.47 \pm 0.472$ & $21.11 \pm 0.522$ & $21.68 \pm 0.611$ & $21.70 \pm 0.668$ & $21.81 \pm 0.869$ & $21.12 \pm 1.319$ \\
HSYA high dose & $20.90 \pm 0.572$ & $21.17 \pm 0.539$ & $22.09 \pm 0.610$ & $21.88 \pm 0.601$ & $22.35 \pm 0.721$ & $22.31 \pm 0.574$ \\
HSYA medium dose & $20.83 \pm 0.699$ & $21.26 \pm 0.704$ & $21.89 \pm 0.948$ & $21.68 \pm 0.836$ & $22.02 \pm 0.885$ & $21.84 \pm 0.773$ \\
HSYA low dose & $20.74 \pm 0.654$ & $20.59 \pm 0.749$ & $21.17 \pm 0.815$ & $20.78 \pm 0.722$ & $20.75 \pm 0.428$ & $21.18 \pm 0.464$ \\
Cisplatin & $20.55 \pm 0.430$ & $20.48 \pm 0.573$ & $19.96 \pm 0.486^{\mathrm{b}}$ & $18.94 \pm 0.403^{\mathrm{b}}$ & $17.57 \pm 0.479^{\mathrm{b}}$ & $17.93 \pm 0.818^{\mathrm{b}}$ \\
Combination & $20.63 \pm 0.942$ & $20.96 \pm 1.176$ & $20.71 \pm 1.358^{\mathrm{a}}$ & $19.81 \pm 1.462^{\mathrm{b}}$ & $19.07 \pm 1.746^{\mathrm{b}}$ & $17.68 \pm 1.582^{\mathrm{b}}$ \\
\end{tabular}

The control group was treated with $0.2 \mathrm{ml}$ normal saline for 11 days. For 11 days, following the injection of tumor cells, the model group was treated with $0.2 \mathrm{ml}$ normal saline, the HSYA high-dose group was treated with $0.2 \mathrm{ml} 2.25 \mathrm{mg} / \mathrm{kg}$ HSYA, the HSYA medium-dose group was treated with $0.2 \mathrm{ml} 1.13 \mathrm{mg} / \mathrm{kg}$ HSYA and the HSYA low-dose group was treated with $0.2 \mathrm{ml} 0.57 \mathrm{mg} / \mathrm{kg}$ HSYA. The cisplatin group was treated with $0.2 \mathrm{ml} 5 \mathrm{mg} / \mathrm{kg}$ cisplatin and the combination group was treated with $0.2 \mathrm{ml} 0.57 \mathrm{mg} / \mathrm{kg} \mathrm{HSYA}+2.5 \mathrm{mg} / \mathrm{kg}$ cisplatin for 11 days following the injection of tumor cells. ${ }^{\mathrm{a}} \mathrm{P}<0.05$, combination group vs. cisplatin group; ${ }^{\mathrm{b}} \mathrm{P}<0.01$, treatment group vs. model group. HSYA, hydroxyl safflower yellow A.
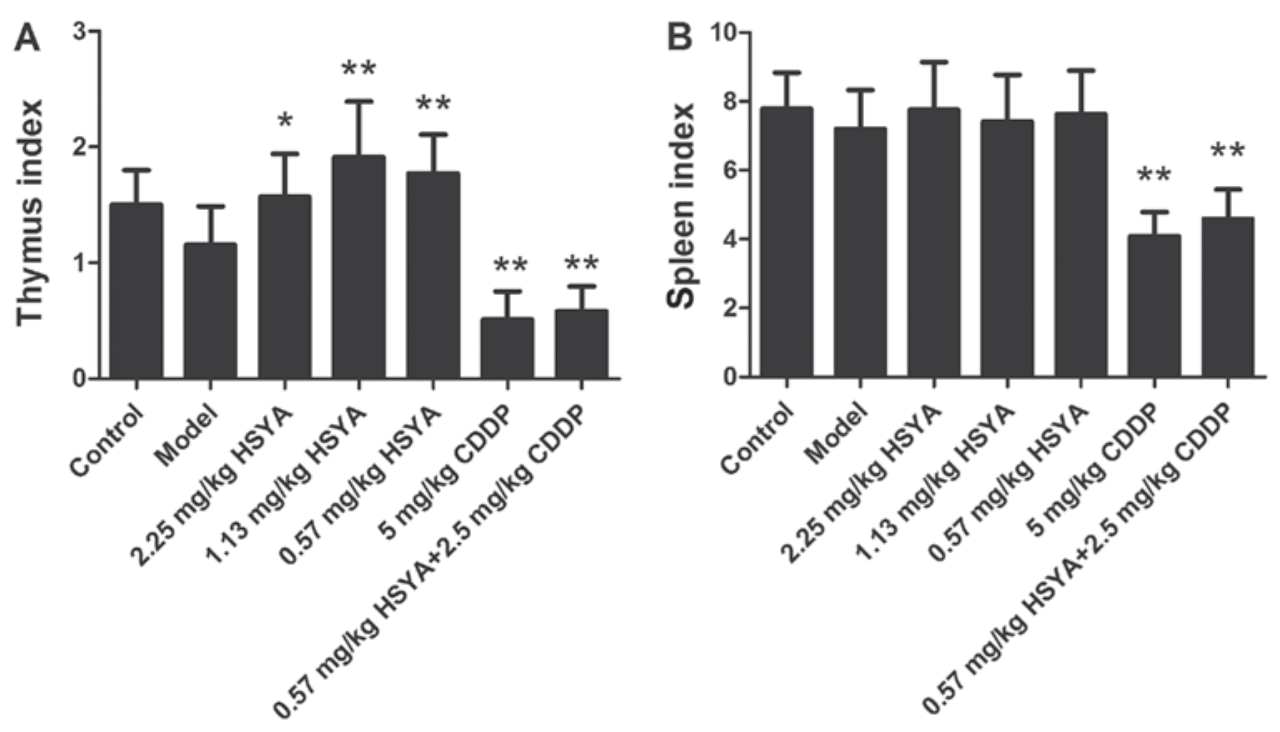

Figure 2. Effect of HSYA treatment on thymus and spleen indexes in hepatocellular carcinoma model mice. (A) Effect of HSYA and/or cisplatin on thymus index. (B) Effect of HSYA and/or cisplatin on spleen index. "P $<0.05$ and ${ }^{* *} \mathrm{P}<0.01$ vs. model group. HSYA, hydroxyl safflower yellow A; CDDP, cisplatin.

A
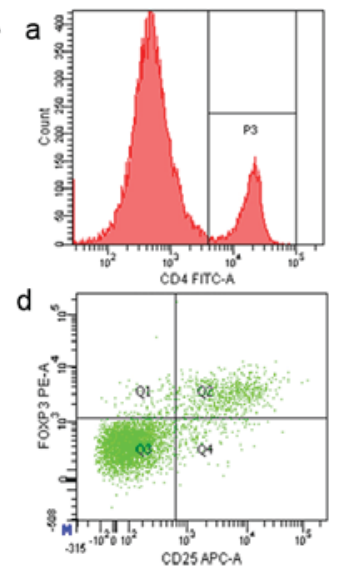
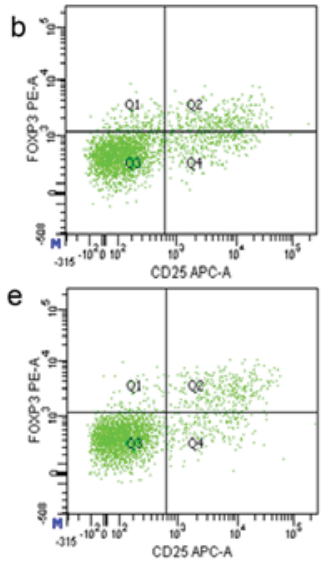
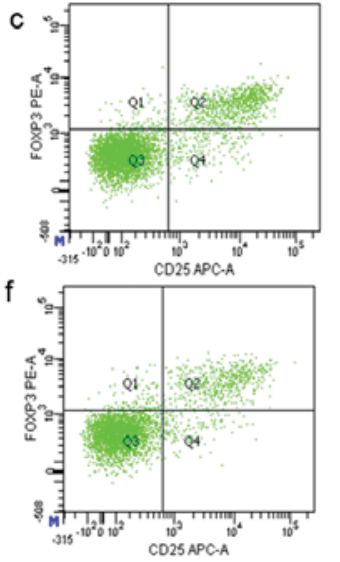

B

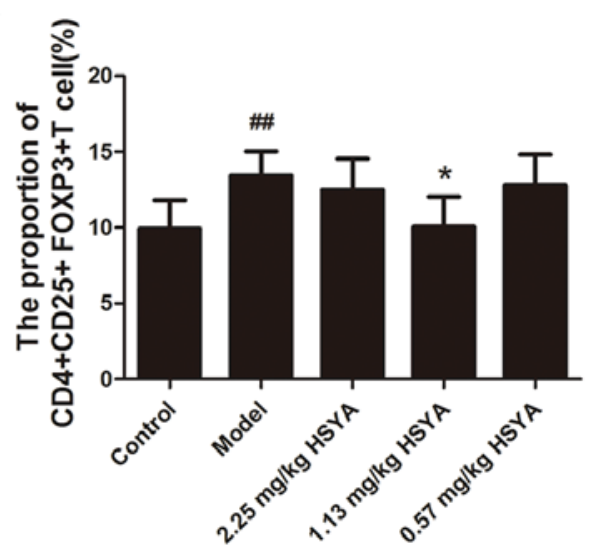

Figure 3. Effect of HSYA on the proportion of $\mathrm{CD}^{+}{ }^{+} \mathrm{CD} 25^{+} \mathrm{FOXP} 3^{+}$Tregs in the spleens of hepatocellular carcinoma model mice. (A) Representative flow cytometric profiles of $\mathrm{CD}^{+} \mathrm{CD} 25^{+} \mathrm{FOXP} 3^{+}$Tregs in the spleen. (Aa) and (Ab) control group; (Ac) model group; (Ad) $2.25 \mathrm{mg} / \mathrm{kg} \mathrm{HSYA}$; (Ae) $1.13 \mathrm{mg} / \mathrm{kg}$ HSYA; (Af) $0.57 \mathrm{mg} / \mathrm{kg}$ HSYA. (B) Proportion of $\mathrm{CD} 4{ }^{+} \mathrm{CD} 25^{+} \mathrm{FOXP} 3^{+}$cells vs. $\mathrm{CD} 4{ }^{+}$cells. ${ }^{*} \mathrm{P}<0.05$ vs. model group; ${ }^{\#} \mathrm{P}<0.01$ vs. control group. HSYA, hydroxyl safflower yellow A; CD4, T-cell surface glycoprotein CD4; CD25, IL-2 receptor subunit $\alpha$ CD25; FOXP3, forkhead box P3; Tregs, regulatory T cells; FITC-A, fluorescein isothiocyanate; PE-A, phycoerythrin; APC-A, allophycocyanin. 

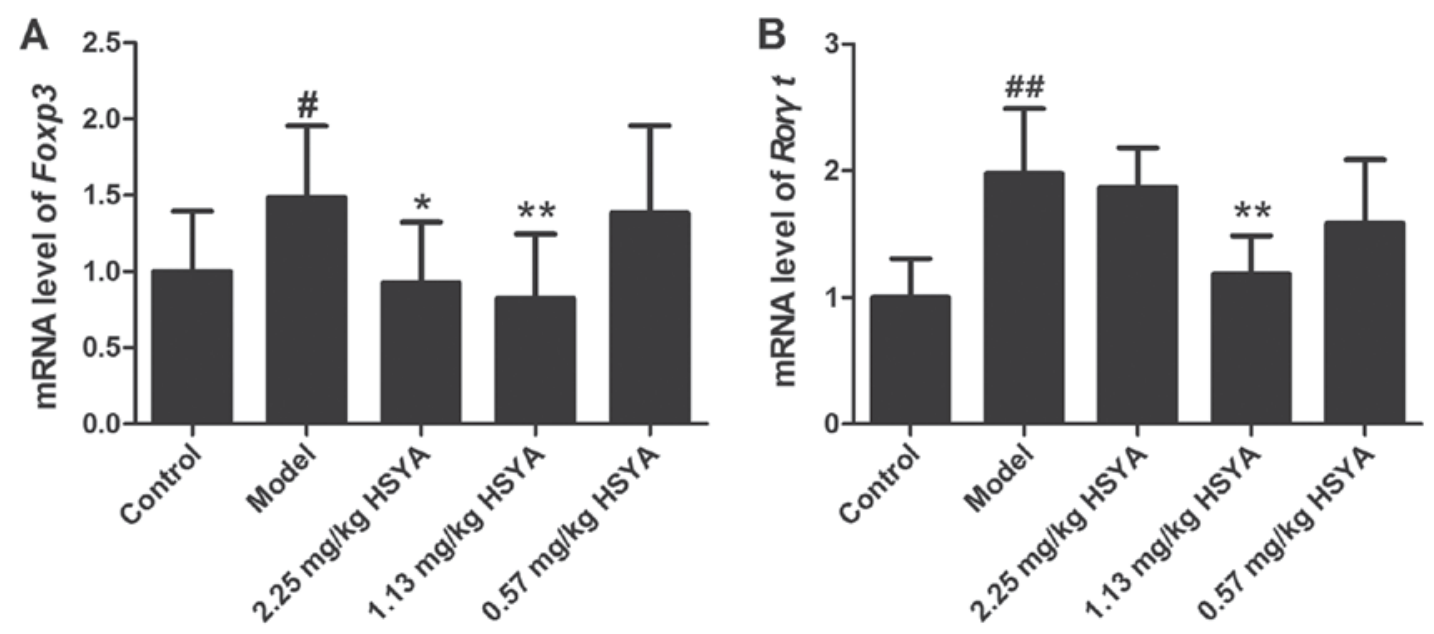

Figure 4. Effect of HSYA on the mRNA expression of Foxp3 and Ror $\gamma t$ in hepatocellular carcinoma model mice. (A) Effect of HSYA treatment on the expression of Foxp 3 mRNA. (B) Effect of HSYA treatment on the expression of Ror $\gamma t$ mRNA. Samples from spleens of the mice were used for reverse transcription-quantitative polymerase chain reaction. $\beta$-actin was used for the normalization of mRNA expression. ${ }^{\#} \mathrm{P}<0.05$ and ${ }^{\# \#} \mathrm{P}<0.01$ vs. control group; ${ }^{*} \mathrm{P}<0.05$ and ${ }^{* *} \mathrm{P}<0.01$ vs. model group. HSYA, hydroxyl safflower yellow A; Foxp3, forkhead box P3; Ror $\gamma t$, retinoic acid receptor-related orphan receptor- $\gamma$.

FOXP3

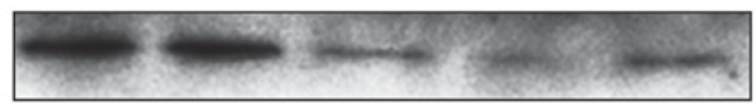

ROR $\gamma t$
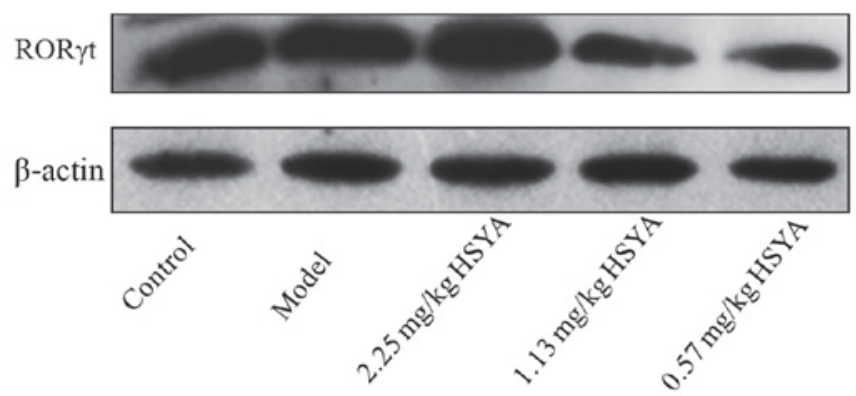

Figure 5. Effect of HSYA on FOXP3 and ROR $\gamma$ t protein expression in hepatocellular carcinoma model mice. Control mice were treated with $0.2 \mathrm{ml}$ normal saline for 11 days; model mice received the same treatment subsequent to an injection of Hepa1-6 cells; in the other groups, mice were treated with doses as shown subsequent to the injection of Hepa1-6 cells Tissue from the tumors of the mice were used for western blotting. HSYA, hydroxyl safflower yellow A; FOXP3, forkhead box P3; ROR $\gamma$ t, retinoic acid receptor-related orphan receptor- $\gamma$ protein.

in the model group, the expression of FOXP3 protein in the HSYA groups was decreased, which was most evident for the middle-concentration group (1.13 mg/kg) (Fig. 5). The middleand low-concentration groups also induced a reduction in ROR $\gamma t$ protein expression. These results indicated that HSYA treatment decreases the expression of FOXP3 and ROR $\gamma t$ protein, two important modulators of the tumor immune microenvironment. Therefore, HSYA treatment may improve the tumor immune microenvironment of HCC model mice.

\section{Discussion}

HSYA has been demonstrated to protect cardiovascular, cerebrovascular and nerve cells, and to prevent inflammation, oxidation and tumors (30-33). As aforementioned, cisplatin is one of the most commonly used cancer chemotherapy drugs. Cisplatin inhibits DNA replication in cancer cells and damages the structure of cancer cell membranes, but its harmful side effects limit its use. Studies have revealed that combining traditional Chinese medicine with cisplatin may enhance its antitumor effect $(34,35)$. Park et al (36) found that cisplatin combined with HemoHIM, a preparation of three herbs, not only significantly reduced tumor volumes, but also enhanced the activity of interferon- $\gamma$ and IL-2, and increased the ratio of cytotoxic T cells and natural killer cells in the spleen of mice. The spleen and thymus are important organs for immune surveillance. Therefore, the spleen and thymus indexes are two important indicators for the effect of HSYA on the immune system of mice (37). An increasing index indicates enhanced immunity (28).

In the present study, the pathological results demonstrated that the number of cancer cells was reduced following treatment with HSYA, with the effect of $1.13 \mathrm{mg} / \mathrm{kg}$ being the most apparent, indicating that HSYA inhibited tumor growth most effectively at this concentration. In the cisplatin and HSYA combined therapy group, the number of cancer cells was reduced more than in the cisplatin alone group, and the extent of liver injury was reduced, indicating that HSYA could enhance the anticancer effect of cisplatin, and reduce liver tissue damage due to cisplatin chemotherapy. The body weight and thymus index of mice receiving HSYA tended to increase compared with the model group, while mice receiving cisplatin exhibited a reduced weight, thymus and spleen index. This indicated that cisplatin treatment has a negative effect on the physical condition of mice and their immune system, whereas HSYA did not affect the weight of HCC model mice. Moreover, at a certain concentration, HSYA improved the immunity of HCC model mice, as well as reducing cisplatin chemotherapy-induced weight loss.

Tregs inhibit antitumor immune responses and FOXP3 is an inhibitory transcription factor within them (38). It was previously illustrated that Tregs are enriched in HCC tissue (39). Another previous study demonstrated that the proportion of $\mathrm{FOXP}^{+}$Tregs in tumor tissue and the proportion of $\mathrm{CD} 4^{+} \mathrm{CD} 25^{+}$Tregs/CD4 ${ }^{+} \mathrm{T}$ lymphocytes in the spleen were negatively correlated with patient prognosis. Therefore, there is clinical value in measuring FOXP3 ${ }^{+}$Tregs to predict 
HCC recurrence and survival (40). Reducing the proportion of Tregs may inhibit tumor growth by regulating the immunosuppressive state of the tumor microenvironment (41).

Th17 cells are a key component of the inflammatory and immune responses that produce IL-17. In HCC patients, the presence of Th17 cells is negatively associated with the overall and recurrence-free survival rate (42). Huang et al (43) found that intrahepatic IL-17 ${ }^{+}$Th17 cells and FOXP3 ${ }^{+}$Tregs exhibited a synergistic effect, promoting the progress of HCC. ROR $\gamma \mathrm{t}$ is a Th17 cell-specific transcription factor (13). A previous study found that the mRNA levels of $R O R \gamma t$ and FOXP3 were significantly increased in peripheral blood mononuclear cells from patients with HCC, indicating high Th17 and Treg numbers (44).

The results of the present study demonstrated that $1.13 \mathrm{mg} / \mathrm{kg}$ HSYA significantly reduced the proportion of Tregs in the spleens of HCC model mice, and reduced the expression of Foxp3 and Ror $\gamma t$ in mouse cancer tissue, indicating that HSYA may suppress tumor growth and immune escape by reducing the number of Tregs, potentially reducing inflammation to improve the tumor immune microenvironment and relieving the immunosuppressive state. The specific mechanisms of this effect remain to be studied.

In summary, $1.13 \mathrm{mg} / \mathrm{kg}$ HSYA regulated the immune microenvironment of $\mathrm{HCC}$ model mice by reducing the proportion of Tregs in the spleen, and the expression of Foxp3 and Ror $\gamma t$ in tumor tissue, enhancing the immunity of mice and reducing the side effects of cisplatin when used at a particular concentration, thus exerting an anticancer effect. However, the immune environment of the body is complex, with a variety of immune factors, and the present study selected a small portion of them to investigate. The effect of HSYA on other important immune factors requires further examination.

\section{Acknowledgements}

The authors would like to thank Dr Wenbin Ou (Institution of Life Sciences, ZheJiang Sci-Tech University), who assisted with the experimental design and data analysis.

\section{Funding}

The present study was supported by the National Natural Science Foundation of China (grant no. 81473655, 31500640 and 31770849), the Beijing University of Traditional Chinese Medicine Graduate Student Project (grant no. 2015-JYB-XS041), the Natural Science Foundation of Zhejiang Province (grant no. LY15C070002 and LY16C050001), the fund from the Science Technology Department of Zhejiang Province (grant no. 2015C33131 and 2016F10005) and the fund from the Science and Technology Bureau of Jiaxing (grant no. 2015AY23007).

\section{Availability of data and materials}

All data generated or analyzed during this study are included in this published article.

\section{Authors' contributions}

YM and CF analyzed the data and wrote the manuscript. JW, ZC and PW performed the experiments. AF, XW, XY, DG, HX and
LL assisted in preparing the experiments. QZ and XL designed the study. All authors have read and approved the final manuscript.

\section{Ethics approval and consent to participate}

The present study was approved by the Institutional Animal Ethics Committee of Beijing University of Chinese Medicine.

\section{Patient consent for publication}

Not applicable.

\section{Competing interests}

The authors declare that they have no competing interests.

\section{References}

1. Yang JD, Nakamura I and Roberts LR: The tumor microenvironment in hepatocellular carcinoma: Current status and therapeutic targets. Semin Cancer Biol 21: 35-43, 2011.

2. Luo P, Yin P, Hua R, Tan Y, Li Z, Qiu G, Yin Z, Xie X, Wang X, Chen W, et al: A Large-scale, multicenter serum metabolite biomarker identification study for the early detection of hepatocellular carcinoma. Hepatology: 28 Sep, 2017 (Epub ahead of print).

3. Parkin DM, Bray F, Ferlay J and Pisani P: Global cancer statistics, 2002. CA Cancer J Clin 55: 74-108, 2005.

4. Ferlay J, Shin HR, Bray F, Forman D, Mathers C and Parkin DM: Estimates of worldwide burden of cancer in 2008: GLOBOCAN 2008. Int J Cancer 127: 2893-2917, 2010.

5. Chen JG: Trends in the incidence of liver cancer and its primary prevention in China. J Clin Hepatol 28: 256-260, 2012.

6. Bruix J and Sherman M; American Association for the Study of Liver Diseases: Management of hepatocellular carcinoma: An update. Hepatology 53: 1020-1022, 2011.

7. Karaman B, Battal B, Sari S and Verim S: Hepatocellular carcinoma review: Current treatment, and evidence-based medicine. World J Gastroenterol 20: 18059-18060, 2014.

8. Greten TF, Wang XW and Korangy F: Current concepts of immune based treatments for patients with HCC: From basic science to novel treatment approaches. Gut 64: 842-848, 2015.

9. Mbeunkui F and Johann DJ Jr: Cancer and the tumor microenvironment: A review of an essential relationship. Cancer Chemother Pharmacol 63: 571-582, 2009.

10. Fritz JM, Dwyer-Nield LD and Malkinson AM: Stimulation of neoplastic mouse lung cell proliferation by alveolar macrophage-derived, insulin-like growth factor-1 can be blocked by inhibiting MEK and PI3K activation. Mol Cancer 10: 76, 2011.

11. Liu J, Zhang H, Jia L and Sun H: Effects of Treg cells and IDO on human epithelial ovarian cancer cells under hypoxic conditions. Mol Med Rep 11: 1708-1714, 2015.

12. Mathai AM, Kapadia MJ,Alexander J,Kernochan LE,Swanson PE and Yeh MM: Role of Foxp3-positive tumor-infiltrating lymphocytes in the histologic features and clinical outcomes of hepatocellular carcinoma. Am J Surg Pathol 36: 980-986, 2012.

13. Castro G,Liu X,Ngo K, De Leon-Tabaldo A,Zhao S,Luna-Roman R, Yu J, Cao T, Kuhn R, Wilkinson P, et al: ROR $\gamma$ t and ROR $\alpha$ signature genes in human Th17 cells. PLoS One 12: e0181868, 2017.

14. Liao R, Sun J, Wu H, Yi Y, Wang JX, He HW, Cai XY, Zhou J, Cheng YF, Fan J and Qiu SJ: High expression of IL-17 and IL-17RE associate with poor prognosis of hepatocellular carcinoma. J Exp Clin Cancer Res 32: 3, 2013.

15. Zhang Q, Zheng J, Wang L, et al: Therapeutic effect of Ramosetron on preventing gastrointestinal reaction induced by chemotherapeutic drugs including Cisplatin in 80 cases. Chin Pharm 10: 1024-1025, 2007.

16. Liu ZQ, Cheng JT, Zhu HX, et al: Experimental study on increasing effect and decreasing its side-effect of Cisplatin of LiuJunZi decoction. Lishizhen Medicine and Materia Medical Research 20: 2492-2494, 2009.

17. Wu H, Lv SZ, Ying XZ, Ye YL and Tian ZF: Effect of traditional Chinese medicine in immunity recovery of radiotherapy in patients with liver cancer. Chin Archiv Trad Chin Med 8: 1997-1999, 2015 (In Chinese). 
18. Wu QL, Lu HD, Guo XL and Kong QZ: Exploration of treatment of malignant tumors with Chinese herbs that invigorate the blood and remove stasis. J Int Trad Western Med 5: 530-531, 2010.

19. Zhou X, Tang L, Xu Y, Zhou G and Wang Z: Towards a better understanding of medicinal uses of Carthamus tinctorius L. in traditional Chinese medicine: A phytochemical and pharmacological review. J Ethnopharmacol 151: 27-43, 2014.

20. Wang J, Wang P, Gui S, Li Y, Chen R, Zeng R, Zhao P, Wu H, Huang $\mathrm{Z}$ and $\mathrm{Wu}$ J: Hydroxysafflor yellow $\mathrm{A}$ attenuates the apoptosis of peripheral blood CD4+ T lymphocytes in a murine model of sepsis. Front Pharmacol 8: 613, 2017.

21. Xie H, Zhang Q, Zhao YQ, Cui W and Niu X: The experiment of safflor yellow effecting the cell growth cycle of mouse $\mathrm{H} 22$ grafting tumor. China J Tradit Chin Med Pharm: 171-173, 2006 (In Chinese).

22. Ma L, Liu L, Ma Y, Xie H, Yu X, Wang X, Fan A, Ge D, Xu Y, Zhang $\mathrm{Q}$ and Song C: The role of E-cadherin/ $\beta$-catenin in hydroxysafflor yellow A inhibiting adhesion, invasion, migration and lung metastasis of hepatoma cells. Biol Pharm Bull 40 : 1706-1715, 2017.

23. Xi S, Zhang Q, Xie H, Liu L, Liu C, Gao X, Zhang J, Wu L, Qian L and Deng X: Effects of hydroxy safflor yellow A on blood vesse and mRNA expression with VEGF and bFGF of transplantation tumor with gastric adenocarcinoma cell line BGC-823 in nude mice. Zhongguo Zhong Yao Za Zhi 34: 605-610, 2009 (In Chinese)

24. Wang J, Zhang Q, Gu LG, Cui W, Xie H and Niu XY: Effect of hydroxy safflor yellow A on the cell cycle and apoptosis of human umbilical vein endothelial cells with the stimulus of tumor cell conditioned medium. J Beijing Univ Tradit Chin Med 31: 741-744, 2008 (In Chinese).

25. Xi SY, Zhang Q, Liu CY, et al: Effects of hydroxy safflower yellow A on the expression of bFGF protein and MMP-9 in human gastric cancer xenografts. Chin J Chin Materia 21: 2877-2881, 2010 (In Chinese).

26. Xi SY, Zhang Q, Liu CY, Xie H, Yue LF, Li WD, Zang BX and Gao XM: Effects of HSYA on protein and mRNA expression of KDR, HIF-1 $\alpha$ and protein expression of VEGF in nude mice with BGC-823 transplantation tumor. Chin J Trad Chin Med Pharm 27: 82-87, 2012.

27. Limani P, Borgeaud N, Linecker M, Tschuor C, Kachaylo E, Schlegel A, Jang JH, Ungethüm U, Montani M, Graf R, et al: Selective portal vein injection for the design of syngeneic models of liver malignancy. Am J Physiol Gastrointest Liver Physiol 310: G682-G688, 2016.

28. Lu YM and Zhang H: Effects of electroacupuncture on T-lymphocytes, spleen index, thymus index and lymphopoiesis levels in strenuous exercise-induced stress rat. Zhen Ci Yan Jiu 37: 136-139, 2012.

29. Livak KJ and Schmittgen TD: Analysis of relative gene expression data using real-time quantitative PCR and the 2(-Delta Delta C(T)) method. Methods 25: 402-408, 2001

30. Dong F, Xue C, Wang Y, Peng Y, Zhang Y, Jin M and Zang B: Hydroxysafflor yellow A attenuates the expression of inflammatory cytokines in acute soft tissue injury. Sci Rep 7: 40584, 2017.

31. Min J and Wei C: Hydroxysafflor yellow A cardioprotection in ischemia-reperfusion (I/R) injury mainly via Akt/hexokinase II independent of ERK/GSK-3 $\beta$ pathway. Biomed Pharmacother 87: 419-426, 2017.
32. Wang T, Duan SJ, Wang SY, Lu Y, Zhu Q, Wang LJ and Han B: Coadministration of hydroxysafflor yellow A with levodopa attenuates the dyskinesia. Physiol Behav 147: 193-197, 2015.

33. Sun L, Yang L, Xu YW, Liang H, Han J, Zhao RJ and Cheng Y: Neuroprotection of hydroxysafflor yellow A in the transient focal ischemia: Inhibition of protein oxidation/nitration, 12/15-lipoxygenase and blood-brain barrier disruption. Brain Res 1473: 227-235, 2012.

34. Wu XL: Efficacy enhancing effect of Shikonin on human liver cancer HepG2 cells treated by Cisplatin. Chin Med J Res Prac 31: 35-38, 2017 (In Chinese).

35. Wu Y and Liu R: Study on the drug-resistant reversal effects of ginsenoside Rh2 in human hepatocellular carcinoma HepG2/ADM cells and its mechanism. J Med Postgra 30: 476-480, 2017.

36. Park HR, Ju EJ, Jo SK, Jung U, Kim SH and Yee ST: Enhanced antitumor efficancy of cisplatin in combination with HemoHIM in tumor-bearing mice. BMC Cancer 9: 85, 2009.

37. Liu Y, Jing Y, Guo LL, et al: Effects of Guben Zhike Recipe on the spleen index, thymus index and Th1/Th2 unbalance in COPD mice. China J Tradit Chin Med Pharm, 2015 (In Chinese).

38. Fontenot JD, Rasmussen JP, Williams LM, Dooley JL, Farr AG and Rudensky AY: Regulatory T cell lineage specification by the forkhead transcription factor foxp3. Immunity 22: 329-341, 2005.

39. Fu J, Xu D, Liu Z, Shi M, Zhao P, Fu B, Zhang Z, Yang H, Zhang $\mathrm{H}$, Zhou $\mathrm{C}$, et al: Increased regulatory $\mathrm{T}$ cells correlate with CD8 T-cell impairment and poor survival in hepatocellular carcinoma patients. Gastroenterology 132: 2328-2339, 2007.

40. Lin SZ, Chen KJ, Xu ZY, Chen H, Zhou L, Xie HY and Zheng SS: Prediction of recurrence and survival in hepatocellular carcinoma based on two Cox models mainly determined by FoxP3+ regulatory T cells. Cancer Prev Res (Phila) 6: 594-602, 2013.

41. Takeuchi $\mathrm{Y}$ and Nishikawa $\mathrm{H}$ : Roles of regulatory $\mathrm{T}$ cells in cancer immunity. Int Immunol 28: 401-409, 2016.

42. Zhang JP, Yan J, Xu J, Pang XH, Chen MS, Li L, Wu C, Li SP and Zheng L: Increased intratumoral IL17-producing cells correlate with poor survival in hepatocellular carcinoma patients. J Hepatol 50: 980-989, 2009.

43. Huang Y, Wang F, Wang Y, Zhu Z, Gao Y, Ma Z, Xu R and Du Z: Intrahepatic interleukin-17+ $\mathrm{T}$ cells and FoxP3+ regulatory $\mathrm{T}$ cells cooperate to promote development and affect the prognosis of hepatocellular carcinoma. J Gastroenterol Hepatol 29: 851-859, 2014.

44. Lin ZW, Wu LX, Xie Y, Ou X, Tian PK, Liu XP, Min J, Wang J, Chen RF, Chen YJ, et al: The expression levels of transcription factors T-bet, GATA-3, ROR $\gamma$ t and FOXP3 in peripheral blood lymphocyte (PBL) of patients with liver cancer and their significance. Int J Med Sci 12: 7-16, 2015. 